\title{
CHANGES ON THORACIC AORTA'S STRUCTURE WITH AN ANTIARRHYTHMIC AGENT
}

\author{
Frade-Mochales M., Pazó-Sayós L., Delgado-Martos MJ., Fernández-Criado C., Delgado- \\ Baeza E., Quintana-Villamandos B. \\ Department of Anaesthesiology and Critical Care ; General University Hospital Gregorio Marañón. Madrid \\ Medicine Faculty. Complutense University, Madrid. Medicine Faculty. Autónoma University, Madrid.
}

\section{BACKGROUND AND GOAL OF STUDY}

Dronedarone is a multichannel blocker used to treat atrial fibrillation (AF). Left hypertrophy is widely known as AF substrate. Our group previously proved that treatment with dronedarone produces an early regression of myocardial remodelling in an experimental model of arterial hypertension ${ }^{1}$. Thus, we wondered if it could also induce changes on Aorta's structure.

\section{MATERIAL AND METHODS}

Adult male spontaneously hypertensive rats (SHR) were randomly divided into therapy group (SHR-D, $n=8$ ) and placebo group (SHR, $n=8$ ). Wistar Kyoto rats were used as normotensive controls (WKY $n=8$ ). After 2 weeks of intervention, systolic blood pressure (SBP) and heart rate (HR) were measured and ascending thoracic aorta was dissected to study its histological characteristics (a total of 8 segments of thoracic aorta per group were stained with orcein and analized): internal and external diameters (ID, ED), cross-sectional area (CSA), wall width (WW). Comparisons among groups were made by ANOVA test of one factor with Bonferroni's correction. $\mathrm{P}<0.05$ was considered statistically significant. All procedures were approved by the Ethics Committee of Hospital General Universitario Gregorio Marañón, Madrid, Spain.-

\begin{tabular}{|c|c|c|c|c|c|c|c|}
\hline \multicolumn{8}{|c|}{ RESULTS } \\
\hline & $\begin{array}{l}\text { WKY } \\
(n=8)\end{array}$ & $\begin{array}{c}\text { SHR } \\
(n=8) \\
\end{array}$ & $\begin{array}{l}\text { SHR-D } \\
(n=8)\end{array}$ & $\mathbf{v}$ & $\begin{array}{l}\text { WKY } \\
(n=8)\end{array}$ & $\begin{array}{c}\text { SHR } \\
(n=8)\end{array}$ & $\begin{array}{l}\text { SHR-D } \\
(n=8)\end{array}$ \\
\hline Weight(g) & $457.21 \pm 2$ & $398.83 \pm 8^{* *}$ & $372.36 \pm 4^{* * *}$ & ED $(\mathrm{mm})$ & $1.76 \pm 0.01$ & $2.32 \pm 0.02^{* * *}$ & $2.16 \pm 0.01^{* * *,+++}$ \\
\hline & & & & ID (mm) & $1.50 \pm 0.01$ & $1.83 \pm 0.01^{* * *}$ & $1.84 \pm 0,01^{* * *}$ \\
\hline HR (bpm) & $399 \pm 11$ & $408 \pm 15$ & $286 \pm 13^{* * *,+++}$ & & & & \\
\hline & & & & WW mm & $0.12 \pm 0.009$ & $0.24 \pm 0.009^{* * *}$ & $0.16 \pm 0.007^{*+++}$ \\
\hline $\begin{array}{l}\text { SBP } \\
(\mathrm{mmHg})\end{array}$ & $134 \pm 10$ & $183 \pm 11^{* *}$ & $143 \pm 14^{++}$ & CSA mm² & $0.65 \pm 0.04$ & $1.57 \pm 0.07^{* * *}$ & $1.01 \pm 0.04^{* * *,+++}$ \\
\hline
\end{tabular}

Table 1. ${ }^{* * P} P<0.01$ vs. WKY; ${ }^{* * * P}<0.001$ vs. WKY; ${ }^{++} P<0.01$

Table 2. Structure of thoracic aorta. ${ }^{* * * P}<0.001$ vs. WKY; ${ }^{++} P<0.001$ vs. $S H R ;{ }^{+++} P<0.001$ vs. SHR. Values presented: mean \pm SEM.

vs. SHR. Values presented as mean \pm SEM. External Diameter (ED), Internal Diameter (ID), Wall Width (WW), Cross-Sectional Area (CSA)
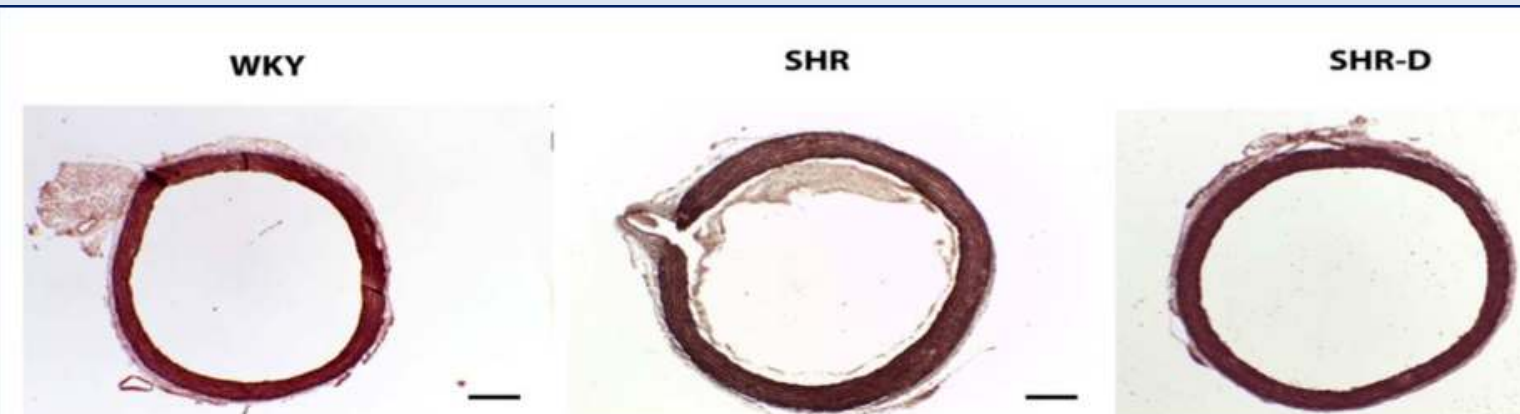

Figure 1- Thoracic aorta's structure (orcein stained)

Only fourteen days of treatment with dronedarone produced a regression in aortic structural remodelling of spontaneously hypertensive rats. 